\title{
A novel automatic classification system based on hybrid unsupervised and supervised machine learning for electrospun nanofibers
}

\author{
Cosimo Ieracitano, Annunziata Paviglianiti, Maurizio Campolo, Amir Hussain, Eros Pasero \\ and Francesco Carlo Morabito
}

\begin{abstract}
The manufacturing of nanomaterials by an electrospinning process requires accurate and meticulous inspection of Scanning Electron Microscope (SEM) images of the electrospun nanofiber, to ensure no structural defects are produced. The possible presence of anomalies is known to make the nanofibrous material useless in the practical application of any nanotechnology. Hence, automatic monitoring and quality control of nanomaterials has become an important challenge in the context of Industry 4.0. In this paper, we propose a novel automatic classification system for homogenous (anomaly-free) and nonhomogenous (with defects) nanofibers avoiding the processing of the redundant full SEM image. Specifically, the image to be analyzed is partitioned into sub-images (nanopatches) that are then used as input to a hybrid unsupervised and supervised machine learning system. An Autoencoder (AE) is first trained with unsupervised learning to generate a code representing the input image with a number of relevant features. Next, a Multilayer Perceptron (MLP), trained with supervised learning, uses the extracted features to classify non-homogenous nanofiber (NH-NF) and homogenous nanofiber (H-NF) materials. The resulting novel AE-MLP system is shown to outperform other standard machine learning models and recent state-of-the-art techniques, reporting accuracy rates up to $\mathbf{9 2 . 5 \%}$. In addition, our proposed approach achieves significant model complexity reduction with respect to other deep learning strategies such as Convolutional Neural Networks (CNN). The promising performance achieved in this benchmark study will stimulate the application of our proposed framework in a range of challenging industrial manufacturing tasks.
\end{abstract}

Index Terms-Material Informatics, Nanomaterials, Electrospinning, Machine Learning, Autoencoder, Anomaly detection.

\section{INTRODUCTION}

In recent years, nanostructured materials have gained continuously growing scientific and industrial interest, because of their versatile applications. Nanotechnology combined with information and communication technology (ICT) represents the frontier of the fifth industrial revolution: because of their reduced size may allow for automation of tasks, which were previously inaccessible due to physical restrictions [1]. Nanomaterials shows the capability to work at the molecular

C. Ieracitano, M. Campolo and F. C. Morabito are with DICEAM, University Mediterranea of Reggio Calabria, Via Graziella, Feo di Vito, 89060 Reggio Calabria, Italy (email: cosimo.ieracitano@unirc.it, campolo@unirc.it, morabito@unirc.it). Corresponding Author: C. Ieracitano

A. Paviglianiti and E. Pasero are with Politecnico di Torino, DET, 10129 Turin, Italy (email:annunziata.paviglianiti@polito.it, eros.pasero@polito.it ).

A. Hussain is with School of Computing, Edinburgh Napier University, Edinburgh EH10 5DT, Scotland, UK (email: a.hussain@ napier.ac.uk). level, creating large structures with versatile properties, which can improve quality of everyday life in different areas [2]. In biomedical field, the huge progress of nanomaterials research suggests that they could yield interesting alternatives to healthcare solutions. In tissue engineering application, nanofibers are used for the reproduction of tissue architecture on the nanoscale, thus giving an impulse to wearable applications for monitoring and remote control. The use of nanofiber materials act as an excellent structure for adhesion, proliferation and cells scaffolding differentiation for musculoskeletal tissue engineering, skin tissue engineering, vascular tissue engineering, neural tissue engineering and as potential vectors for the controlled delivery of proteins and DNA [3]. Among many nanofibers synthesis techniques, electrospinning appears to be the most promising technology to meet these industrial objectives. Electrospun fibers can indeed be applied to study drug delivery, encapsulating the therapeutic agent in the fibers and maintaining the integrity and bioactivity of molecules due to slight processing parameters. Indeed, the release of the drug depends on the degradation of the polymer fibers. Hence, it can be adequately controlled. In bioengineering, the nanofibers could allow to include substrate-based optical antenna systems for improved bio-sensing applications [4]. Because of their mechanical, thermodynamic, acoustic, optical, electrical and magneto-electric properties, they are also applied in chemical engineering for water quality improvement problems [5] [6]. Nanofibers can yield good membranes in environmental engineering systems due their novel properties like high porosity. In this context, electrospinning membranes are emerging as a versatile technique with promising features for water treatment. Nanotechnology has exceptional potential for filtration applications due to its ability to create structurally controlled materials [7]. In renewable energy, nanofibers are used as polymer solid electrolytes for battery applications. These polymers are selected due to their properties like low density, easy fabrications and low chemical corrosion. Nanomaterials are used widely for energy storage devices due to their high surface areas and porosities. Nanofiber solid electrolytes, produced by electrospinning, can be also used in developing lithium ion batteries, fuel cells, dye-sensitized solar cells and supercapacitors [8].

Recently, Nanoelectronics has emerged as a novel approach to produce electronic component with peculiar lectrical and electro-optical properties that stem from the metals and metal composites. Nanofiber applications success requires to play 
special care to the quality of nanomaterial and thus to the generation process: the regular arrangement of the material grid and the absence of irregularities ensures the optimization of the nanomaterial properties. In particular, the Electrospinning method is based on the electrostatic force of a polymeric solution, which causes the generated drop to fall towards a conductive collector, where the polymeric nanomaterial settles. Material quality, nanomaterial diameter size and the presence of anomalies (i.e, beads and flattened areas) depend on the selected electrospinning processing parameters: i.e., voltage level, tip-to-collector distance, diameter of the needle, feed rate and type of collector [9].

Nanotechnology and nanomaterials promise to generate products characterized by unprecedented and enhanced properties, obtainable by changing the microscopic structure of materials rather than through their processing on a macroscopic scale. In principle, the products can have industrial and commercial relevance, particularly for the approach to the so-called Industry 4.0. Industry 4.0 looks at the industries as smart factories that aim to increase productivity and reduce production costs by integrating information and communication technology. Industry 4.0 focuses specifically on automation, i.e. the use of techniques and methodologies that increase the production quality of the industrial system [10] [11]. In nanomaterial production, a crucial step to practical implementation of automation is the defect identification process, in order to reduce the number of laboratory experiments and the burden of the experimentation phase. The idea here proposed is to design, by suitable training, a model capable of emulating the recognition process carried out by the operators, subject to fatigue in the visual inspection. There are different types of anomalies that can affect the industrial process, in particuler: beads, i.e., agglomerates of material whose diameter is significantly larger than the rest of the fiber; films, i.e., thin layers of polymer that lies on nanofibers and holes, i.e., large dark areas not covered by nanofibers.

In this paper, a detection method that prescinds from the specific defect is proposed. In order to detect anomalies in nanomaterials, a machine learning (ML) approach is investigated. ML algorithms extract mathematical models based on experimentally generated training data that are used to make predictions on novel instances of the emulated phenomenon. The data here used (i.e., nanoimages) have been generated through a Scanning Electron Microscope (SEM), at the Materials for Environmental and Energy Sustainability Laboratory of the University Mediterranea of Reggio Calabria (Italy). The selected ML scheme is a deep neural network whose scope is to discriminate between homogenous $(\mathrm{H}-$ $\mathrm{NF}$ ) and non-homogenous nanofibers (NH-NF) by inspecting sub-patches of the generated SEM images. NH-NF will be automatically discarded during the inspection. Fig. 1 shows the presentation of two different types of nanomaterials, the former characterized by homogeneous nanofibers, the latter by non-homogeneous nanofibers.

The neural network topology used for solving the defect detection and classification problem is based on a cascade of an autoencoder (AE), trained with an unsupervised rule, and a standard multilayer perceptron (MLP) trained with backpropagation. This series of AE and MLP composes the proposed hybrid unsupervised and supervised ML system. It is worth mentioning that the $\mathrm{AE}$ has the capability to learn an efficient and compact data representation through a code learned by exploiting the available data without using the associated label, thus extracting reliable features from unlabeled data. Here, SEM images are compressed by means of the developed AE and the corresponding decoding stage reconstructs an approximation of the original patch. Specifically, the unsupervised processor, represented by the AE, generates a latent representation of the input SEM image by compressing it into a code; then, the decoding stage approximately reconstruct the original image on the basis of the extracted features; the supervised processor, represented by the MLP, performs the probabilistic decision on the quality of the image (i.e., $\mathrm{NH}$ $\mathrm{NF}$ or H-NF) using the features previously extracted by the AE.

The original contribution of this paper is to address the problem of classification without using the redundant full image generated by the microscope but subdividing them in patches and then compressing each sub-part generating a code that allows distinguishing defective from non-defective patches with a strongly reduced computational capacity. This will facilitate the training of the classification system also improving the generalization to new experiments.

The rest of this paper is structured as follows: in Section II, the related works and the state-of-the-art on ML approaches for nanomaterial classification is presented. In Section III, the electrospinning process is described including the generation of the database. Section IV introduces the hybrid unsupervised and supervised methodology here proposed; in Section V the achieved results are shown; in Section VI, conclusions and future work are presented.

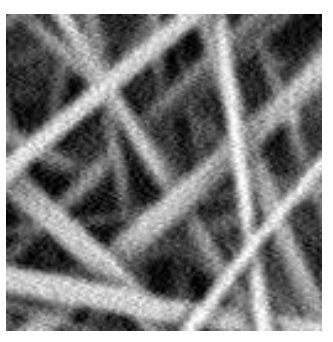

(a)

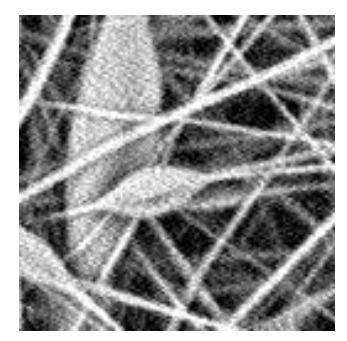

(b)

Fig. 1. Examples of SEM images of nanofibrous materials: (a) without defects and (b) with a defective bead. 


\section{RELATED WORKS}

During the past decade, nanomaterials have gained understanding because of the multiple potential applications. The recognition of the materials quality implies an acceleration in the production chain for the use of nanomaterials in the industrial sector. The use of ML-based recognition techniques has accordingly received growing interest in automation. Conventional defect detection algorithms based upon image structure features mainly detects the surface defects by analyzing the texture, skeleton, edge and spectrum of the image. In [12], spatial correlation functions defined between the bands of a sensor are used to recognize the color structure. A linear model for surface spectral reflectance, is used to show that changes in illumination and geometry correspond to a linear transformation of both the correlation functions and their coordinates. In [13] is presented an approach to gray scale and rotation invariant texture classification based on local binary patterns and nonparametric discrimination of sample and prototype distributions. Another technique for automatic defect inspection is based on the selection of a suitable threshold. A commonly used threshold technique is the Otsu method that exploits a bimodal distribution histogram [14]. As a consequence, the method fails when the histogram of an image is unimodal. For this reason, the weighted object variance method (WOV) is proposed. It is able to detect defects on the surfaces, by means of a parameter that is equivalent to the cumulative probability of the occurrence of defects; it is weighted on the variance between classes [15] [16]. Another approach to defect recognition is a combination of image segmentation method and heat map technique. Image segmentation has an important impact on industry artificial vision; its goal is to divide the images in different salient portions of the image, which give feedback about the presence of defects. The heat map is a spreadsheet that contains colors instead of numbers. The default color gradient sets the lowest value in the heat map to dark blue, the highest value to bright red and the mid-range values to light gray. Heat maps can be used to identify matrix portions with similar values, since they are displayed as areas of similar color, favoring the anomaly detection. Thus, defects in the original input image can be detected automatically after processing the heat map with a threshold segmentation method. It is possible to perform simultaneously the classification and localization of defects [17] . In order to reduce production times and increase production quality, processes were automated through machine learning (ML) and, in particular, deep learning (DL), algorithms where lot of data are available to extract experience from them. DL is an approach to Artificial Intelligence (AI), that achieves great power by representing data as a nested hierarchy of concepts, starting from simpler concepts to more abstract representations [18] [19]. DL has been successfully employed in several applications, such us in cyber security [20] [21], in neuro-science [22] [23] [24], in sentiment classification [25] [26], image decomposition [27] and fault detection systems [28]. DL has also been proposed to deal with the problem of automatic detection of surface anomalies. In [29], a multilayer feed forward network trained with back propagation algorithm has been applied for weld defects identification. However, DL techniques are the most performing architectures designed for detection and segmentation of surface anomalies. Most of the above-mentioned methods are based on image processing that implies detecting defect contours with strong contrast and low noise, at certain scales, or under specific illumination conditions. Convolutional neural network (CNN) is an advanced method to classify images [30]. With regards to the automatic nanomaterial anomalies detection in SEM images, some works have been proposed in the recent literature. Boracchi et al. addresses the issue of automatic detection of anomalies in SEM images, allowing an intelligent system to control independently the validity of the data acquired with a sparse-based model representations [31]. Carrera et al. implemented a dictionary-based algorithm that learns a model yielding sparse representations of the structures that characterize correctly produced nanomaterials [32]. Napoletano et al. presented a region-based method for detection and anomaly localization in SEM images, based on CNN self-similarity. The degree of anomaly is assessed by means of a CNN, considering a dictionary generated from anomalies-free subimages belonging to a training set [33]. The implementation of DL for the automatic detection of defects has also been addressed by Ieracitano et al. [34] [35]. In particular, a CNN has been proposed to automatically classify the SEM images of H-NF and NH-NF, interpreted as two different categories. As a difference with most of the previous papers, in this work both samples with and without anomalies are analyzed. This approach appears more significant as the images are typically generated with different sets of configuration parameters, which implies a variety of possible ranges of presentation for the nanofibers also in absence of anomalies. However, being it a fully data-driven approach, it is data hungry, requiring the collection of lot of examples through suitably designed laboratory experiments. In this paper, a DL architecture is designed for segmenting nanoimages and localizing defects. In particular, an AE neural network transforms the input image into a feature vector which is used as a prediction mask. The experimental results demonstrate that this method meets the robustness and accuracy requirements while reducing the computational burden and suggesting a data augmentation technique.

\section{Experimental Set-up AND Dataset CONSTRUCTION}

\section{A. Electrospinning process}

Electrospinning is the most versatile process for nanofibers fabrication as it is characterized by the ability to improve tissue performance allowing specific modifications for each type of application [36]. The nanofiber fabrication method requires an instrumental apparatus (see Fig. 2) that is comprised of a highvoltage supply, an extruder and a grounded metallic collector screen where the fibers are collected. A polymeric solution is initially contained into a dosing syringe, regulated by the volumetric pump, which allows controlling the flow-rate. A high-voltage is applied between the needle of the syringe (anode) and the collector (cathode), which are electrostatically 
charged to a different electric potential. By increasing the applied voltage, the surface charge of the polymeric solution increases while the radius of the polymeric solution drop decreases, until a critical voltage value. At this moment, the drop takes the form of a cone, referred to as Taylor cone [37]. Due to the electric field, a jet (consisting of only a nanofiber), from the cone to the collector, is generated; meanwhile the solvent evaporates and is deposited on the collector in the form of nanofibers. Viscosity, electrical conductivity and surface tension of the polymer solution affect the diameter and the morphology of the generated fibers [38]. Specifically, increasing the viscosity also increases the diameter of the fibers, because the solution opposes more resistance to the elongation by the electric field, and consequently the jet stabilizes and makes a shorter path. The increase of the electrical conductivity of the solution causes a greater repulsion of charge jet, and a higher ironing of the fibers, which decrease in diameter. Therefore, in order to produce the nanofibers, the electrical charge imposed must exceed the surface tension of the solution. It is therefore advisable to have a solution with low surface tension.

1) Materials, experimental setup and dataset construction: The main parameters used in electrospinning to control the morphology of the nanofibers for the generation of samples are Concentration $\left(p_{1}\right)$, applied voltage $\left(p_{2}\right)$, flow rate $\left(p_{3}\right)$ and tip-to-collector distance TCD $\left(p_{4}\right)$. For the laboratory experiments here carried out, a CH-01 Electrospinner 2.0 (Linari Engineering s.r.l.) was used with a $20 \mathrm{~mL}$ glass syringe, equipped with a stainless steel needle of $40 \mathrm{~mm}$ length and 0.8 $m m$ thick. Polyvinylacetate (PVAc) as polymer and Ethanol $(\mathrm{EtOH})$ as solvent compose the solution used in this study. To obtain a homogeneous polymer solution, it was placed in a test tube and then in a magnetic stirrer, a tool used to mix solvent and solute, by rotating a magnetic latch. To analyze the signals out of the interaction of the beam with the nanomaterial produced by electrospinning process, the Phenom Pro-X scanning electron microscope (SEM) was used. It is an electro-optical instrument based on the emission of an electron beam. After the material production, the Fibermetric SEM images analyzer was used to evaluate the average diameter, the distribution of the nanofibers and the presence of anomalies (i.e., structural defects). Sixteen experiments were carried out at different working conditions at the Materials for Environmental and Energy Sustainability Laboratory of the Mediterranea University of Reggio Calabria, Italy), as reported in Table I. It is to be noted that the experiments were developed by varying the aforementioned parameters $\left(p_{1}, p_{2}\right.$, $\left.p_{3}, p_{4}\right)$ in the well defined working range: $p_{1}[10 ; 25] \% w t ; p_{2}$ $[10 ; 17.5] \mathrm{kV} ; p_{3}[100 ; 300] \mu L / m i ; p_{4}[10-15] \mathrm{cm}$. The $e^{t h}$ nanofibrous material (with $e=1,2, . .16$ ), underwent to the SEM analizer and 10 relevant and representative areas were selected by an expert operator. Hence, a total of $16 \times 10=160$ SEM images (sized $128 \times 128$ ) were collected [34]. However, as the ultimate goal of the present study is to address the problem of classification without using the redundant full image generated by the microscope, each SEM image (sized 128 x 128) was partitioned into four patches (here referred as to nanopatches) of the same size 64 × 64 (as shown in Fig. 3). Each SEM patch was manually classified by the nanomaterials expert in two different classes: H-NF and NH-NF images. It is worth mentioning that homogeneous nanomaterial fabrication is typically observed with high values of voltages and concentrations; while non-homogenous nanomaterial fabrication are affected by the presence of anomalies, such us beads or films, that can occur when the polymeric solution is made up with low values of concentrations or when the tip-to-collector distance is too high.

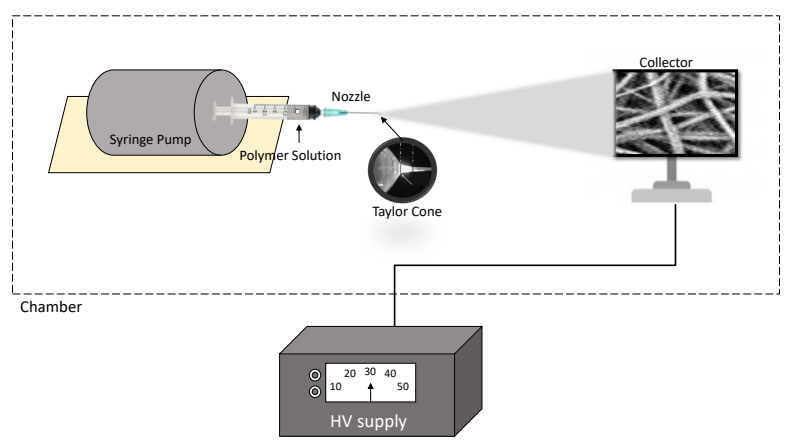

Fig. 2. A typical layout of the electrospinning apparatus.

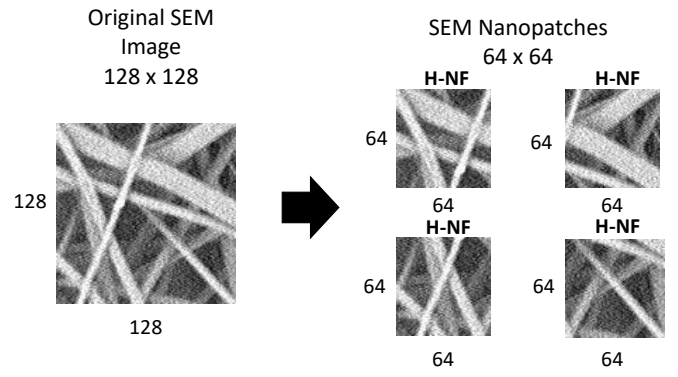

Fig. 3. Example of a SEM image sized $128 \times 128$ partitioned into four SEM nanopatches sized $64 \times 64$. Note that, in this case, all the sub-images belog to the homogeneous nanofiber (H-NF) class.

TABLE I

SETUP OF THE ELECTROSPINNING EXPERIMENTS.

\begin{tabular}{|c|c|c|c|c|}
\hline$\#$ & $\begin{array}{c}\text { Concentration } \\
\left(\boldsymbol{p}_{1}\right)[\% w t]\end{array}$ & $\begin{array}{c}\text { Voltage } \\
\left(\boldsymbol{p}_{2}\right)[\mathrm{kV}]\end{array}$ & $\begin{array}{c}\text { Flow rate } \\
\left(\boldsymbol{p}_{3}\right)[\mu L / \mathrm{min}]\end{array}$ & $\begin{array}{c}\text { TDC } \\
\left(\boldsymbol{p}_{4}\right)[\mathrm{cm}]\end{array}$ \\
\hline 1 & 10 & 15 & 10 & 100 \\
\hline 2 & 15 & 10 & 10 & 100 \\
\hline 3 & 15 & 13.5 & 10 & 100 \\
\hline 4 & 15 & 15 & 10 & 100 \\
\hline 5 & 15 & 15 & 10 & 200 \\
\hline 6 & 15 & 15 & 10 & 300 \\
\hline 7 & 15 & 15 & 12.5 & 100 \\
\hline 8 & 15 & 15 & 13.5 & 100 \\
\hline 9 & 15 & 15 & 15 & 100 \\
\hline 10 & 20 & 10 & 10 & 100 \\
\hline 11 & 20 & 11.5 & 10 & 100 \\
\hline 12 & 20 & 13.5 & 10 & 100 \\
\hline 13 & 20 & 15 & 10 & 100 \\
\hline 14 & 20 & 16 & 10 & 100 \\
\hline 15 & 20 & 17.5 & 10 & 100 \\
\hline 16 & 25 & 15 & 10 & 100 \\
\hline
\end{tabular}




\section{HYBRID UNSUPERVISED AND SUPERVISED MACHINE LEARNING SYSTEM}

Fig. 4 shows the architecture of the proposed hybrid unsupervised and supervised machine learning system for SEM images produced by electrospinning procedure. Specifically, the proposed system includes two main modules: the unsupervised processor (Fig. 4a), i.e., an Autoencoder (AE) that performs the features extraction operation; the supervised processor (Fig. 4b), i.e., a Multilayer Perceptron (MLP) that performs the classification task: NH-NF vs. H-NF. The processing modules are extensively detailed in the following Sections.

\section{A. Autoencoder}

Autoencoders (AEs) are neural networks trained with unsupervised learning technique that are commonly used for the tasks of representation learning and dimensionality reduction [39] [40]. The most typical topology includes an encoding and a decoding stage. AEs commonly exploit backpropagation learning algorithm with a suitable cost function with the objective of making the output as similar as possible to the input while building an internal latent representation of reduced size. AEs thus projects the input image into a lower-dimensional hidden layer (called latent-space representation) and then try to reconstruct the output from this reduced representation. After the compression phase, the number of neurons of the hidden layer should be smaller w.r.t. the input layer and the output layer. In the encoding stage, the network is forced to learn the hidden features behind the input data. In the decoding stage, the $\mathrm{AE}$ reconstructs the input layer data at the output layer with optimal accuracy [41]. In this way, the internal representation extracts the most significant aspects (i.e., features) of the image presented at the input by exploiting its redundancy. As shown in Fig. 3, AE works in two steps: an encoder represented by the function $y=f(x)$ and a decoder that generates the reconstruction $z=g(y)$.

In short, AEs can be described by the function:

$$
g(f(x))=z
$$

where $z$ is as close as possible to the original input $x$. The encoder contains the input layer and the hidden layer, where input data is mapped to obtain a deterministic latent-space representation $y$.

$$
y=\sigma\left(W^{T} x+b\right)
$$

where $\sigma$ is typically a sigmoidal or other nonlinear functions; $x$ the input dataset, $W$ represents the encoder's weight matrix and $\mathbf{b}$ is an offset vector. The decoder consists of the hidden layer and the output layer. In this case, the latent space representation is inversely mapped to obtain the output $z$ :

$$
z=\hat{W}^{T} x+\hat{b}
$$

Where $\hat{W}$ is the reconstruction decoder's weights matrix and $\hat{b}$ is the reconstruction offset vector. Finally, in order to reproduce the outputs more and more similar to the inputs, the error function $J(x, z)$ is minimized.

$$
J(x, z)=\frac{1}{2}(x-z)^{2}
$$

The ideal AE should be sensitive enough to the input to build an accurate reconstruction, while, at the same time, insensitive enough to it in order to avoid the model may simply overfit the training data. This tradeoff is achieved by taking advantage of the redundancies of the input [42].

1) Feature extraction: Fig. 4a illustrates the architecture of the proposed AE-based unsupervised processor employed for features extraction. It includes an AE[4096:256:4096]. Notably, given the $n^{\text {th }}$ NH-NF/H-NF SEM sub-image (i.e., nanopatch) sized $64 \times 64$, it is firstly flatten into a vector $1 \mathrm{x}$ 4096. Next, the proposed AE compresses the input representation ( $x$, sized 1 x 4096) into a latent-space ( $y$, sized $1 \mathrm{x} 256)$ subsequently used to decode the same input space $(z \approx x$, sized $1 \times$ 4096). The $\mathrm{AE}$ [4096:256:4096] is trained with unsupervising learning mode for about $10^{3}$ epochs on a workstation Intel(R) Core(TM) i7-8700K CPU @ $3.7 \mathrm{GHz}$ with 64 GB RAM and 1 NVIDIA GeForce RTX $2080 \mathrm{Ti}$ GPU installed (training time $\approx 2 \mathrm{~min}$ ). The hyperbolic tangent is employed for the encoder and the linear function for the decoder module. Note that the hidden layer dimension (1 $x$ 256) of the AE was set after several experimental tests, estimating the minimum reconstruction error. In particular, the minimum mean squared error was of 0.4416 . Hence, overall, a features matrix of $640 \times 256$ (i.e., number of SEM patches $\mathrm{x}$ number of features) was extracted (320 belonging to $\mathrm{NH}-$ $\mathrm{NF}$ and 320 belonging to $\mathrm{H}-\mathrm{NF}$ ). However, since the limited size of such dataset did not allowed to achieve comparable performance on train and test sets, all the features data vectors were corrupted by white Gaussian noise at a $\mathrm{SNR}=10 \mathrm{~dB}$ and included in the original dataset. A grand total of $1280 \times 256$ instances were taken into account (640 belonging to NH-NF and 640 belonging to $\mathrm{H}-\mathrm{NF}$ ).

\section{B. Multilayer Percepetron}

The Multilayer Perceptron (MLP) is the common used feedforward neural network that consist of an input, an output and one or more hidden layers. It belongs to the supervised learning networks that exploit the class label information to minimize a loss function through standard gradient-based backpropagation technique. Each neuron in a MLP is a weighted sum of all its input fed into a non-linear activation function. Fig. 4b shows the proposed MLP. Specifically, the features vector (sized $1 \times 256$ ) previously extracted from the unsupervised processor (i.e., AE) is used as input to a MLP with 2 hidden layers of 100 and 80 hidden units, respectively. Note that the hyperbolic tangent is used as activation function for each hidden neuron. The network ends with a softmax output layer employed to perform the 2-way classification task: NH-NF vs. H-NF. The architecture, here referred to as $\mathrm{MLP}_{100,80}$, was trained over $10^{3}$ epochs on the aforementioned workstation (i.e., Intel(R) Core(TM) i7-8700K CPU @ $3.7 \mathrm{GHz}$ with $64 \mathrm{~GB}$ RAM and 1 NVIDIA GeForce RTX $2080 \mathrm{Ti}$ GPU installed). Training time was on average of about 40 minutes using the leave-one-out technique over the whole dataset. 


\section{Unsupervised-Supervised System}

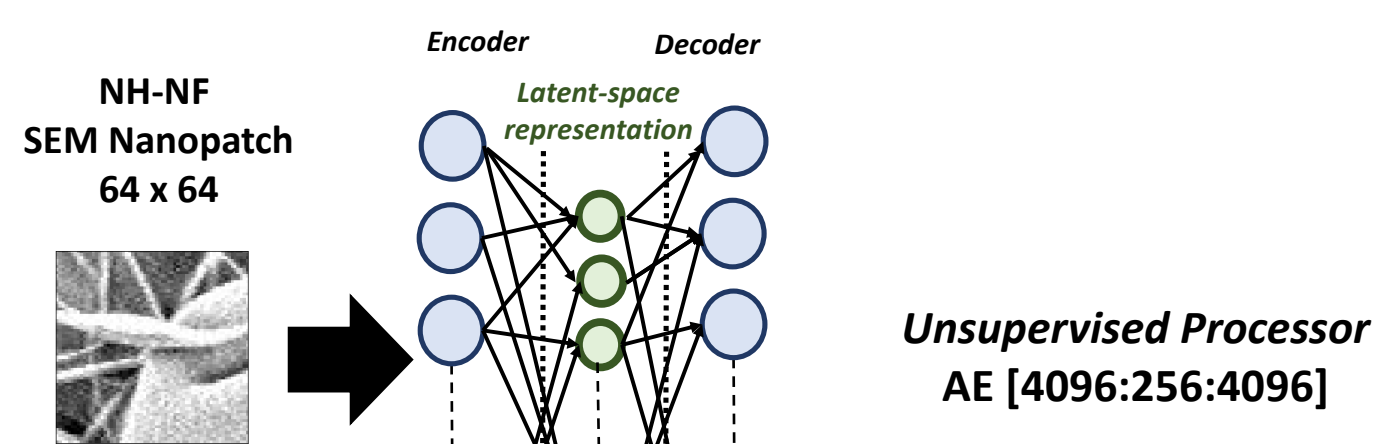

Input

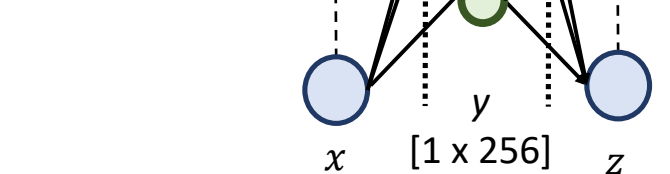

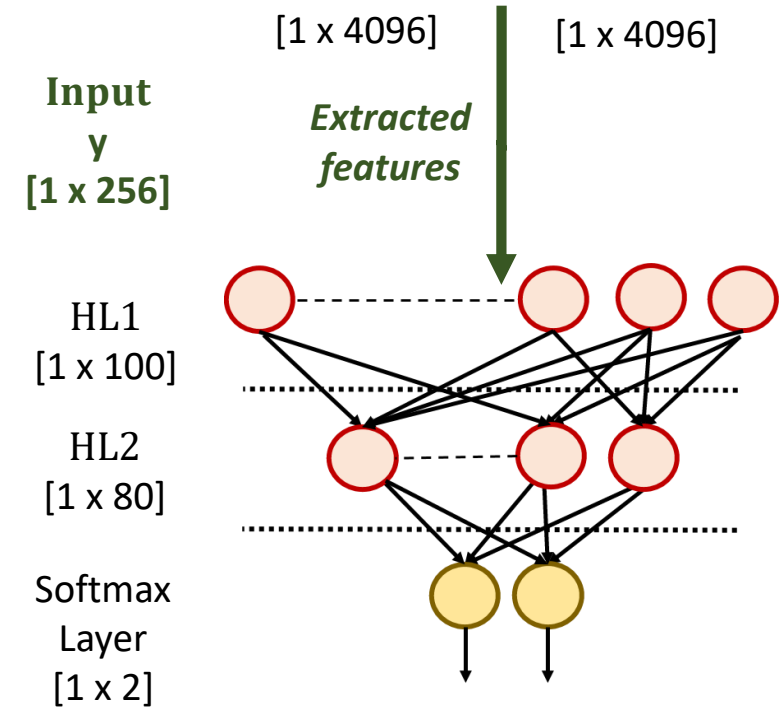

NH-NF H-NF (a)

\section{Supervised Processor MLP [256:100:80:2]}

Fig. 4. Architecture of the proposed hybrid unsupervised and supervised machine learning system. (a) Unsupervised processor composed of an AE [4096:256:4096]. The SEM nanopatch is reshaped into a single vector sized 1 x 4096 and used as input to the proposed AE that allows to extract the most relevant features (sized 1 x 256) from the input data. (b) Supervised processor composed of a MLP [256:100:80:2]. The extracted features are the input to the proposed 2-hidden layers MLP for performing the 2-way classification task: NH-NF vs. H-NF. As an example, in the figure, a NH-NF SEM nanopatch inputs the hybrid unsupervised and supervised classification system.

\section{EXPERIMENTAL RESULTS}

The performance of our proposed hybrid unsupervised and supervised ML system were assessed using the common precision, recall, F-score and accuracy metrics:

$$
\begin{gathered}
\operatorname{Precision}(P r)=\frac{t p}{t p+f p} \\
\operatorname{Recall}(R c)=\frac{t p}{t p+f n} \\
F-\operatorname{score}=2 * \frac{\operatorname{Pr} * R c}{P r+R c}
\end{gathered}
$$

$$
\text { Accuracy }=\frac{t p+t n}{t p+f p+t n+f n}
$$

where $t p, f p, t n, f n$ are true positive, false positive, true negative, false negative, respectively. In this study, tp denotes SEM images with defects correctly identified as NH-NF; $f p$ denotes SEM images of homogeneous nanofibers misclassified as NH-NF; t $n$ is the number of SEM images of homogeneous nanofibers correctly identified as $\mathrm{H}-\mathrm{NF} ; f n$ is the number of SEM images of nanofibers with defects misclassified as H-NF. As described in Section IV, the augmented features dataset of 1280 instances (640 belonging to H-NF and 640 belonging 
to NH-NF) was used as input to our proposed MLP. It is worth mentioning that the leave-one-out technique (LOO) was applied to validate the efficiency and generalization ability of the developed model. Specifically, LOO consists in partitioning repeatedly the dataset into train set, composed of all instances excluded the $i^{t h}$, and test set composed of the $i^{\text {th }}$ left-out observation. Here, the LOO procedure was applied to the whole dataset. Hence, $N=1280$ networks were trained on $\mathrm{N}-1$ data-points and tested on the held-out case.

Note that the best MLP architecture was determined using a trial-and-error approach, namely, estimating the performance of different numbers of hidden neurons and hidden layers. Table II reports comparitive classification performance in terms of precision, recall, F-score and accuracy. First, the 256dimensional input representation was used as input to MLP classifiers with 1-hidden layer of different size. Specifically, 40, 60, 80, 100, 120, 140 hidden units were tested. Experimental results show that the 1-hidden layer MLP with 100 neurons (denoted as $\mathrm{MLP}_{100}$ ) achieved the highest F-score and accuracy: $92.04 \%$ and $91.80 \%$, respectively. Next, additional layers were used in order to find out possible better configurations. In particular, MLP classifiers with 2-hidden layers were tested, that is: $\mathrm{MLP}_{100,40}, \mathrm{MLP}_{100,60}$ and $\mathrm{MLP}_{100,80}$. As can be seen, among these architectures, MLP $_{100,80}$ reported the highest F-score and accuracy: $92.68 \%$ and $92.50 \%$, respectively. Finally, MLP classifiers with 3-hidden layers were tested: $\mathrm{MLP}_{100,80,20}, \mathrm{MLP}_{100,80,40}$ and $\mathrm{MLP}_{100,80,60}$. Here, the higest scores were achieved by MLP $_{100,80,60}$ with Fscore of $90.88 \%$ and accuracy of $90.63 \%$. Hence, comparative results show that the 2-hidden layer MLP $_{100,80}$ achieved the best classification performance in terms of precision $(95 \%)$, recall $(90.48 \%)$, F-score $(92.68 \%)$ and accuracy $(92.50 \%)$.

The proposed MLP 100,80 was also compared with other standard ML techniques. Notably, Support Vector Machine with linear kernel (SVM, [43]) and Linear Discriminant Analysis (LDA, [44]) were developed to perform the 2-way discrimination task (NH-NF vs. H-NF). For fair comparison, LOO procedure was applied to the whole dataset. Table III reports the performance of each classifier evaluated on the test sets: MLP 100,80, SVM and LDA. Specifically, SVM classifier achieved F-score of $65.87 \%$ and accuracy of $66.72 \%$; whereas, LDA classsifier achieved F-score of $64.06 \%$ and accuracy of $64.84 \%$. As can be observed from Table III, our proposed MLP $_{100,80}$ outperformed all of the other models. In support of this outcome, the Receiver Operating Characteristic (ROC) and the corresponding Area Under the Curve (AUC) of the developed MLP, SVM, LDA based classifiers were evaluated. As can be seen in Fig. 5, MLP achieved the highest AUC score of 0.90 .

\section{A. Permutation Analysis}

In order to assess the dependency of the proposed classifier on the available dataset the standard permutation-based $p$ value statistical test is carried out [45]. This test estimates the $p$-value under a certain null hypothesis that is: features and class labels are independent. Specifically, the labels are repeatedly permuted and for each iteration the statistical metric

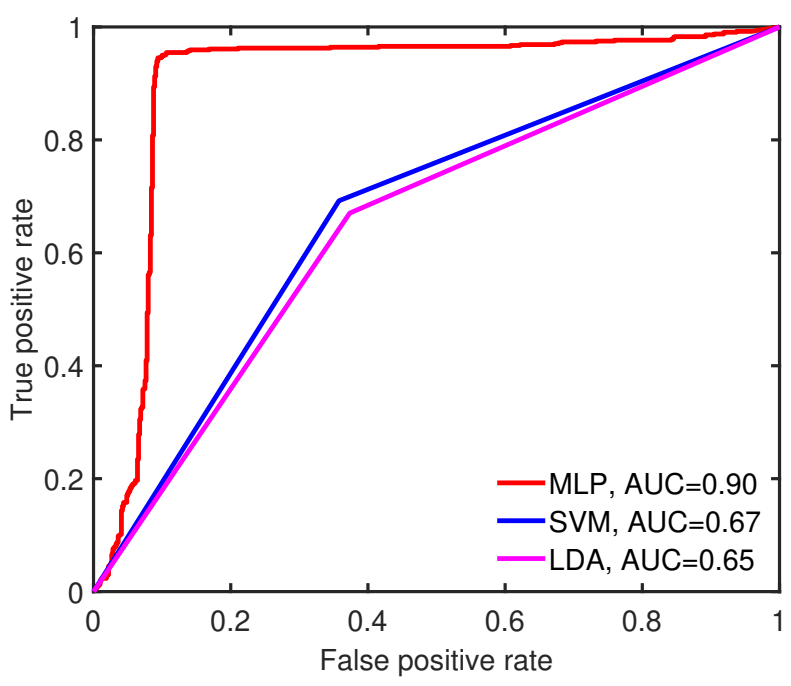

Fig. 5. ROC curves and corresponding AUC values of the proposed MLP, SVM and LDA classifiers for the NH-NF vs. H-NF classification. Note that the figure refers to the best MLP architecture (i.e., $\operatorname{MLP}_{100,80}$ ) as reported in Table III.

of interest (here, the accuracy $A_{i}$, with $\mathrm{i}=1,2, \ldots N_{\text {perm }}$ ) is computed. Finally, $p$-value is empirically calculated as the total number of all $A_{i}$ equal or greater than the performance estimated with the original dataset (i.e, accuracy $A_{o}$ ), divided by the number of permutations $\left(N_{\text {perm }}\right)$. $p$-value smaller than a threshold (typically, $\alpha=0.05$ ) results in rejecting the null hypothesis and consequently concluding that the classifier is statistically significant. It is worth noting that, ideally, all of the possible labels permutations should be taken into account in order the evaluate the exact $p$-value. As this is computationally expensive, in this study, $N_{\text {perm }}=100$ were performed [45]. Experimental results reported that $p$-value $=0.00 / 100=0.00$ $<0.05$. Hence, the null hypothesis is rejected. In conclusion, the proposed classifier is statistically significant.

\section{DISCUSSION}

In this paper, an innovative hybrid unsupervised and supervised ML system is proposed to classify SEM images of electrospun nanofibers. The dataset composed of 160 SEM images of PVAc nanofibrous materials, previously proposed in [34], was here employed. However, in order to address the problem of classification without using the redundant full image generated by the microscope, each SEM image was divided into four nanopatches, resulting in 640 images: 320 belonging to NH-NF and 320 belonging to H-NF. Each SEM image sized $64 \times 64$, it was reshaped into a single vector (sized $1 \times$ 4096) and used as input to the the first module of our proposed hybrid unsupervised and supervised ML system, i.e., the Autoencoder. The developed AE (4096:256:4096) was trained off-line using unsupervised learning (i.e., with no class information) and was employed to automatically extract the most relevant features from the input representation. Next, the compressed 256-dimensional features vector was used as input to the second module of the hybrid unsupervised and supervised ML system, i.e., the Multilayer Perceptron. The 
TABLE II

CLASSIFICATION PERFORMANCE IN TERMS OF PRECISION, RECALL, F-SCORE AND ACCURACY OF MLP WITH DIFFERENT HIDDEN LAYERS (HL) AND HIDDEN UNITS.

\begin{tabular}{|c|c|c|c|c|c|c|c|}
\hline Model & HL1 & HL2 & HL3 & Precision & Recall & F score & Accuracy \\
\hline MLP $_{40}$ & 40 & - & - & $94.06 \%$ & $89.58 \%$ & $91.76 \%$ & $91.56 \%$ \\
\hline MLP $_{60}$ & 60 & - & - & $94.53 \%$ & $88.19 \%$ & $91.25 \%$ & $90.94 \%$ \\
\hline MLP $_{80}$ & 80 & - & - & $94.68 \%$ & $88.85 \%$ & $91.67 \%$ & $91.40 \%$ \\
\hline MLP $_{100}$ & 100 & - & - & $94.84 \%$ & $89.40 \%$ & $92.04 \%$ & $91.80 \%$ \\
\hline MLP $_{120}$ & 120 & - & - & $94.37 \%$ & $89.61 \%$ & $91.93 \%$ & $91.71 \%$ \\
\hline MLP $_{140}$ & 140 & - & - & $92.34 \%$ & $86.03 \%$ & $89.07 \%$ & $88.67 \%$ \\
\hline MLP $_{100,40}$ & 100 & 40 & - & $93.59 \%$ & $89.81 \%$ & $91.66 \%$ & $91.48 \%$ \\
\hline MLP $_{100,60}$ & 100 & 60 & - & $92.03 \%$ & $88.70 \%$ & $90.34 \%$ & $90.16 \%$ \\
\hline $\mathbf{M L P}_{100,80}$ & $\mathbf{1 0 0}$ & $\mathbf{8 0}$ & - & $\mathbf{9 5 \%}$ & $\mathbf{9 0 . 4 8 \%}$ & $\mathbf{9 2 . 6 8 \%}$ & $\mathbf{9 2 . 5 0 \%}$ \\
\hline MLP $_{100,80,60}$ & 100 & 80 & 60 & $93.44 \%$ & $88.46 \%$ & $90.88 \%$ & $90.63 \%$ \\
\hline MLP $_{100,80,40}$ & 100 & 80 & 40 & $91.25 \%$ & $88.75 \%$ & $89.98 \%$ & $89.84 \%$ \\
\hline MLP $_{100,80,20}$ & 100 & 80 & 20 & $90.31 \%$ & $85.76 \%$ & $87.98 \%$ & $87.66 \%$ \\
\hline
\end{tabular}

TABLE III

CLASSIFICATION PERFORMANCE IN TERMS OF PRECISION, RECALL, F-SCORE AND ACCURACY OF THE PROPOSED MLP (I.E., MLP 100,80 ), SVM AND LDA CLASSIFERS

\begin{tabular}{|c|c|c|c|c|}
\hline Model & Precision & Recall & F score & Accuracy \\
\hline MLP $_{100,80}$ & $\mathbf{9 5 \%}$ & $\mathbf{9 0 . 4 8 \%}$ & $\mathbf{9 2 . 6 8 \%}$ & $\mathbf{9 2 . 5 0 \%}$ \\
\hline $\mathrm{SVM}$ & $64.22 \%$ & $67.60 \%$ & $65.87 \%$ & $66.72 \%$ \\
\hline $\mathrm{LDA}$ & $62.66 \%$ & $65.52 \%$ & $64.06 \%$ & $64.84 \%$ \\
\hline
\end{tabular}

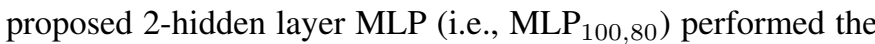
binary discrimination task: NH-NF vs. H-NF.

To the best of our knowledge, this is the first study that deals with the classification of SEM sub-patches limiting the impact of the full image information. Furthermore, the originality of the proposed methodology lies in coding the information of the SEM sub-regions (i.e., lack or presence of defect) into a compressed features vector achieved by the AE processor, from unlabeled data. Such unsupervised data compression allowed to facilitate the supervised training of the classification processor (i.e. MLP). It is worth noting that the average recostruction error of the AE [4096:256:4096] was very small, namely, of only 0.4416 . As an example, Fig. $6 \mathrm{a}$ and 7 report the representation of the 256-dimensional features vector extracted by a H-NF and NH-NF image via the proposed $\mathrm{AE}$. The figures show also the decoded images of 10 H-NF (Fig. 7b) and $10 \mathrm{NH}-\mathrm{NF}$ (Fig. 6b). As can be seen from a visual inspection, the original NH-NF/H-NF image and the corresponding reconstructed NH-NF/H-NF image (produced by the $\mathrm{AE}$ ) are visually similar. Note that the size of $\mathrm{AE}$ was empirically defined after several experimental simulations. Furthermore, in order to find out the best MLP architecture, different numbers of hidden layers and hidden units were also tested (Table II). Experimental results show that our proposed hybrid unsupervised and supervised ML system, that is, the combination of AE-MLP architecures, reported the highest performance when compared with other MLbased classifiers (i.e., SVM, LDA). Specifically, the proposed MLP $_{100,80}$ achived F-score and accuracy rate up to $92.68 \%$ and $92.50 \%$, respectively. Furthermore, a permutation test was carried out to assess the statistical significance of the estimated classification accuracy. For fair comparison, the classification system here presented was also compared with previous works that addressed the classifcation of SEM images (NH-NF vs. H-NF) by using the same dataset of 160 SEM images. Notably, in [34], raw SEM images were fed directly into a deep learning classifier able to achieve accuracy of $80 \%$. However, the developed classification system consisted of a complex and deep CNN with 5 convolutional layers and 5 pooling layers that used the redundant full image information. In [35] SEM images were pre-processed by using an optimized Sobel filtering. The same deep CNN of [34] was employed reporting similar classification performance. In contrast, here, the proposed hybrid unsupervised and supervised ML system allowed to achieve better classification performance (F-score of $92.68 \%$, accuracy of $92.5 \%$ ) using a simpler and lower computationally expensive architecture (consisted of 1-hidden layer $\mathrm{AE}$ and 2-hidden layers MLP). In order to improve the classification abilities of the MLP and to augment the experimental database, in this study, a white Gaussian noise at a $\mathrm{SNR}=10 \mathrm{~dB}$ was used to corrupt the features dataset extracted by the AE. Such noisy feature data were included in the original dataset, increasing its cardinality. This operation allowed to roughly simulate new electrospinning experiments and achieve very good performance. This can also be, in principle, used to emulate experiments at different configuration parameters thus reducing the whole cost of the experimental procedure.

\section{CONCLUSION}

In this paper, we propose a novel automatic classification approach for SEM images of homogenous (anomaly-free) and non-homogenous (with anomalies, namely, structural defect) nanofibres. To this end, a hybrid unsupervised and supervised ML based classification system is developed. Specifically, the combination of an $\mathrm{AE}$ (trained in an unsupervised manner) and a MLP (trained in a supervised manner) is proposed. Experimental simulations show that such a hybrid unsupervised and 
supervised approach achieves the highest performance in terms of precision, recall, F-score, accuracy and AUC. Notably, the proposed AE-MLP sytem outperforms other standard ML techniques as well as recent state-of-the-art methods, reporting an accuracy of up to $92.5 \%$. It is worth mentioning that, in this study, SEM images are subdivided into four patches and analyzed one by one. In the future, a re-assembly step will be carried out: in this case, if at least one quadrant is not homogeneous, the entire SEM image will be classified as not homogeneous and then automatically discarded, through use of fuzzy learning approaches (e.g. [46]). In addition, the proposed unsupervised $\mathrm{AE}$ based methodology can form in principle the basis to a generative model (e.g. [47]) that will allow augmenting the database without the need of carrying out novel costly laboratory experiments. Other novel combinations of state-of-the-art deep and reinforcement [48] and multi-task learning [49] approaches will also be explored for a more extensive comparative evaluation.

\section{ACKNOWLEDGMENT}

The authors would like to thank the researchers of the Materials for Environmental and Energy Sustainability Laboratory at the University Mediterranea of Reggio Calabria (Italy) for providing the SEM image database adopted in this work.

\section{REFERENCES}

[1] S. Rai and A. Rai, "Nanotechnology-the secret of fifth industrial revolution and the future of next generation," Jurnal Nasional, vol. 7, no. 2, pp. 61-66, 2017.

[2] A. Cappy, D. Stievenard, and D. Vuillaume, "Nanotechnology: the next industrial revolution?" 2002.

[3] R. Vasita and D. S. Katti, "Nanofibers and their applications in tissue engineering," International Journal of nanomedicine, vol. 1, no. 1, p. 15 2006.

[4] R. Aliaksandra, "Nanomaterials for biosensing and phototherapy applications," in 2018 International Conference Laser Optics (ICLO). IEEE, 2018, pp. $540-540$.

[5] A.-X. Liu, J.-S. Gao, and M.-Y. Wu, "Effects of nanomaterials on water quality of aquiculture," in 2013 Third International Conference on Intelligent System Design and Engineering Applications. IEEE, 2013, pp. 688-691.

[6] K. M. Yun, C. J. Hogan Jr, Y. Matsubayashi, M. Kawabe, F. Iskandar, and K. Okuyama, "Nanoparticle filtration by electrospun polymer fibers," Chemical Engineering Science, vol. 62, no. 17, pp. 4751-4759, 2007.

[7] I. Tlili and T. A. Alkanhal, "Nanotechnology for water purification: electrospun nanofibrous membrane in water and wastewater treatment," Journal of Water Reuse and Desalination, vol. 9, no. 3, pp. 232-248, 2019.

[8] G. Sun, L. Sun, H. Xie, and J. Liu, "Electrospinning of nanofibers for energy applications," Nanomaterials, vol. 6, no. 7, p. 129, 2016.

[9] P. Ramesh Kumar, N. Khan, S. Vivekanandhan, N. Satyanarayana, A. Mohanty, and M. Misra, "Nanofibers: effective generation by electrospinning and their applications," Journal of nanoscience and nanotechnology, vol. 12, no. 1, pp. 1-25, 2012.

[10] D. Kolberg and D. Zühlke, "Lean automation enabled by industry 4.0 technologies," IFAC-PapersOnLine, vol. 48, no. 3, pp. 1870-1875, 2015.

[11] M. A. K. Bahrin, M. F. Othman, N. N. Azli, and M. F. Talib, "Industry 4.0: A review on industrial automation and robotic," Jurnal Teknologi, vol. 78, no. 6-13, pp. 137-143, 2016.

[12] L. Wang and G. Healey, "Using zernike moments for the illumination and geometry invariant classification of multispectral texture," IEEE Transactions on Image Processing, vol. 7, no. 2, pp. 196-203, 1998.

[13] T. Srikanth, P. P. P. Kumar, and A. Kumar, "Color image segmentation using watershed algorithm," IJCSIT) International Journal of Computer Science and Information Technologies, vol. 2, no. 5, pp. 2332-2334, 2011.
[14] N. Otsu, "A threshold selection method from gray-level histograms," IEEE transactions on systems, man, and cybernetics, vol. 9, no. 1, pp. 62-66, 1979.

[15] X.-c. Yuan, L.-s. Wu, and Q. Peng, "An improved otsu method using the weighted object variance for defect detection," Applied Surface Science, vol. 349, pp. 472-484, 2015.

[16] H.-F. Ng, "Automatic thresholding for defect detection," Pattern recognition letters, vol. 27, no. 14, pp. 1644-1649, 2006.

[17] F. Zhou, G. Liu, F. Xu, and H. Deng, "A generic automated surface defect detection based on a bilinear model," Applied Sciences, vol. 9, no. 15, p. 3159, 2019.

[18] Y. LeCun, Y. Bengio, and G. Hinton, "Deep learning," nature, vol. 521, no. 7553, pp. 436-444, 2015

[19] Y. Bengio, "Deep learning of representations for unsupervised and transfer learning," in Proceedings of ICML workshop on unsupervised and transfer learning, 2012, pp. 17-36.

[20] C. Ieracitano, A. Adeel, F. C. Morabito, and A. Hussain, "A novel statistical analysis and autoencoder driven intelligent intrusion detection approach," Neurocomputing, vol. 387, pp. 51-62, 2020.

[21] H. Zhang, Y. Li, Z. Lv, A. K. Sangaiah, and T. Huang, "A real-time and ubiquitous network attack detection based on deep belief network and support vector machine," IEEE/CAA Journal of Automatica Sinica, 2020.

[22] C. Ieracitano, N. Mammone, A. Bramanti, A. Hussain, and F. C Morabito, "A convolutional neural network approach for classification of dementia stages based on 2d-spectral representation of eeg recordings," Neurocomputing, vol. 323, pp. 96-107, 2019.

[23] T. D. Pham, K. Wardell, A. Eklund, and G. Salerud, "Classification of short time series in early Parkinson' s disease with deep learning of fuzzy recurrence plots," IEEE/CAA Journal of Automatica Sinica, vol. 6 , no. 6, pp. 1306-1317, 2019.

[24] Y. Xia, H. Yu, and F.-Y. Wang, "Accurate and robust eye center localization via fully convolutional networks," IEEE/CAA Journal of Automatica Sinica, vol. 6, no. 5, pp. 1127-1138, 2019.

[25] Z. Wang and Z. Lin, "Optimal feature selection for learning-based algorithms for sentiment classification," Cognitive Computation, vol. 12, no. 1 , pp. 238-248, 2020.

[26] T. Saha, D. Gupta, S. Saha, and P. Bhattacharyya, "Emotion aided dialogue act classification for task-independent conversations in a multimodal framework," Cognitive Computation, pp. 1-13, 2020.

[27] Q. Lian, W. Yan, X. Zhang, and S. Chen, "Single image rain removal using image decomposition and a dense network," IEEE/CAA Journal of Automatica Sinica, vol. 6, no. 6, pp. 1428-1437, 2019.

[28] E. Principi, D. Rossetti, S. Squartini, and F. Piazza, "Unsupervised electric motor fault detection by using deep autoencoders," IEEE/CAA Journal of Automatica Sinica, vol. 6, no. 2, pp. 441-451, 2019.

[29] S. Lalithakumari, B. Sheelarani, and B. Venkatraman, "Artificial neural network based defect detection of welds in tofd technique," International Journal of Computer Applications, vol. 41, no. 20, 2012.

[30] D. Tabernik, S. Šela, J. Skvarč, and D. Skočaj, "Segmentation-based deep-learning approach for surface-defect detection," Journal of Intelligent Manufacturing, vol. 31, no. 3, pp. 759-776, 2020.

[31] G. Boracchi, D. Carrera, and B. Wohlberg, "Novelty detection in images by sparse representations," in 2014 IEEE Symposium on Intelligent Embedded Systems (IES). IEEE, 2014, pp. 47-54.

[32] D. Carrera, F. Manganini, G. Boracchi, and E. Lanzarone, "Defect detection in sem images of nanofibrous materials," IEEE Transactions on Industrial Informatics, vol. 13, no. 2, pp. 551-561, 2016.

[33] P. Napoletano, F. Piccoli, and R. Schettini, "Anomaly detection in nanofibrous materials by cnn-based self-similarity," Sensors, vol. 18, no. 1 , p. 209,2018

[34] C. Ieracitano, F. Pantó, N. Mammone, A. Paviglianiti, P. Frontera, and F. C. Morabito, "Toward an automatic classification of sem images of nanomaterials via a deep learning approach," in Neural Approaches to Dynamics of Signal Exchanges. Springer, 2020, pp. 61-72.

[35] C. Ieracitano, A. Paviglianiti, N. Mammone, M. Versaci, E. Pasero, and F. C. Morabito, "Socnnet: An optimized sobel filter based convolutional neural network for SEM images classification of nanomaterials," in Progresses in Artificial Intelligence and Neural Systems. Springer, p. in press.

[36] Z.-M. Huang, Y. Zhang, S. Ramakrishna, and C. Lim, "Electrospinning and mechanical characterization of gelatin nanofibers," Polymer, vol. 45, no. 15 , pp. 5361-5368, 2004

[37] S. Theron, E. Zussman, and A. Yarin, "Experimental investigation of the governing parameters in the electrospinning of polymer solutions," Polymer, vol. 45, no. 6, pp. 2017-2030, 2004. 


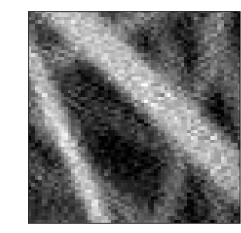

Original H-NF SEM Nanopatch $64 \times 64$

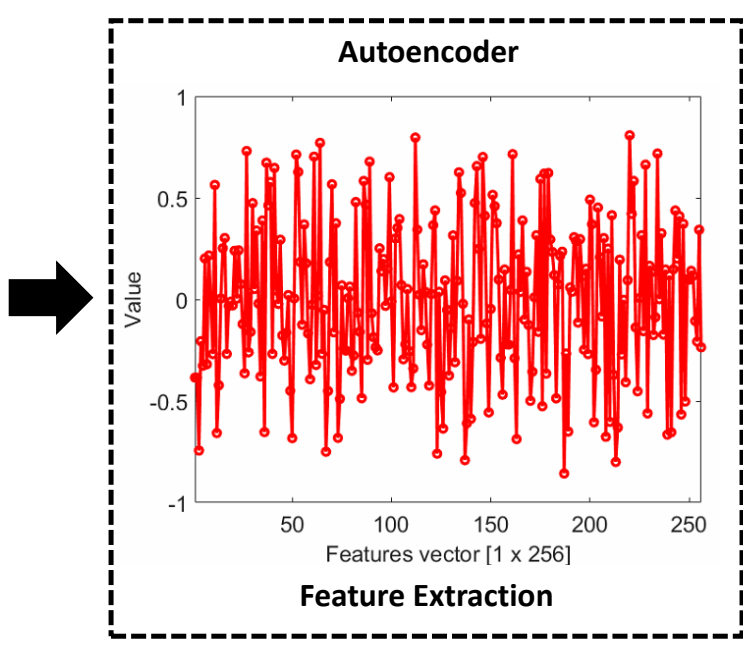

(a)

Original H-NF SEM Nanopatches
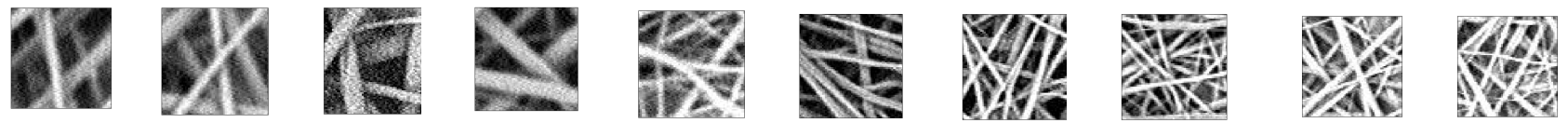

\section{Reconstructed H-NF SEM Nanopatches}
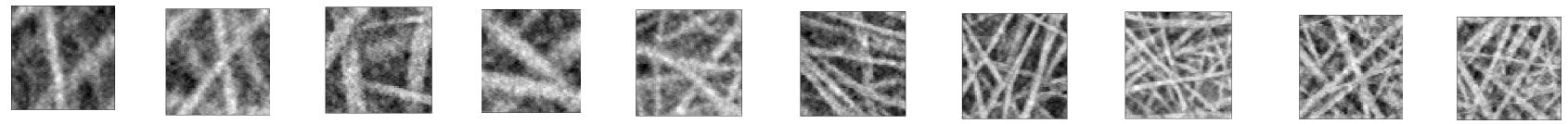

(b)

Fig. 6. (a) Representation of the 256-dimensional features vector extracted by a SEM H-NF image (sized 64 x 64) via the proposed AE. (b) Examples of 10 reconstructed H-NF images.

[38] A. Abutaleb, D. Lolla, A. Aljuhani, H. U. Shin, J. W. Rajala, and G. G. Chase, "Effects of surfactants on the morphology and properties of electrospun polyetherimide fibers," Fibers, vol. 5, no. 3, p. 33, 2017.

[39] B. Du, W. Xiong, J. Wu, L. Zhang, L. Zhang, and D. Tao, "Stacked convolutional denoising auto-encoders for feature representation," IEEE transactions on cybernetics, vol. 47, no. 4, pp. 1017-1027, 2016.

[40] P. Baldi, "Autoencoders, unsupervised learning, and deep architectures," in Proceedings of ICML workshop on unsupervised and transfer learning, 2012, pp. 37-49.

[41] Y. Ollivier, "Auto-encoders: reconstruction versus compression," arXiv preprint arXiv:1403.7752, 2014.

[42] P. Liu, P. Zheng, and Z. Chen, "Deep learning with stacked denoising auto-encoder for short-term electric load forecasting," Energies, vol. 12, no. 12, p. 2445, 2019.

[43] P. Bradley and O. Mangasarian, "Massive data discrimination via linear support vector machines," Optimization methods and software, vol. 13, no. 1 , pp. 1-10, 2000.

[44] S. Balakrishnama and A. Ganapathiraju, "Linear discriminant analysis-a brief tutorial," Institute for Signal and information Processing, vol. 18, pp. 1-8, 1998

[45] M. Ojala and G. C. Garriga, "Permutation tests for studying classifier performance," Journal of Machine Learning Research, vol. 11, no. Jun, pp. 1833-1863, 2010.

[46] A. Alsarhan, Y. Kilani, A. Al-Dubai, A. Zomaya, and A. Hussain, "Novel fuzzy and game theory based clustering and decision making for vanets," IEEE Transactions on Vehicular Technology, 2019.

[47] S. Zhang, K. Huang, R. Zhang, and A. Hussain, "Generalized adversarial training in riemannian space," in 2019 IEEE International Conference on Data Mining (ICDM). IEEE, 2019, pp. 826-835.

[48] M. Mahmud, M. S. Kaiser, A. Hussain, and S. Vassanelli, "Applications of deep learning and reinforcement learning to biological data," IEEE transactions on neural networks and learning systems, vol. 29, no. 6, pp. 2063-2079, 2018.

[49] F. Xiong, B. Sun, X. Yang, H. Qiao, K. Huang, A. Hussain, and Z. Liu, "Guided policy search for sequential multitask learning," IEEE Transactions on Systems, Man, and Cybernetics: Systems, vol. 49, no. 1, pp. 216-226, 2018. 

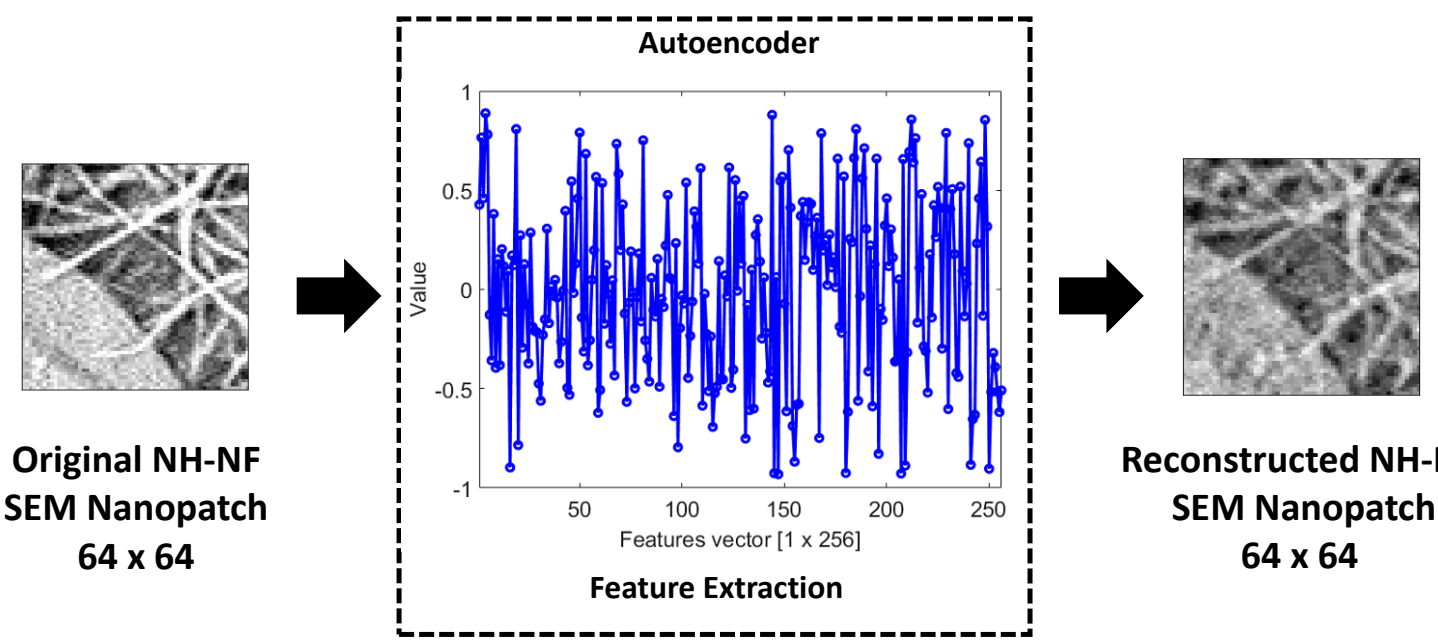

\section{Reconstructed NH-NF \\ SEM Nanopatch}

$64 \times 64$

(a)

Original NH-NF SEM Nanopatches
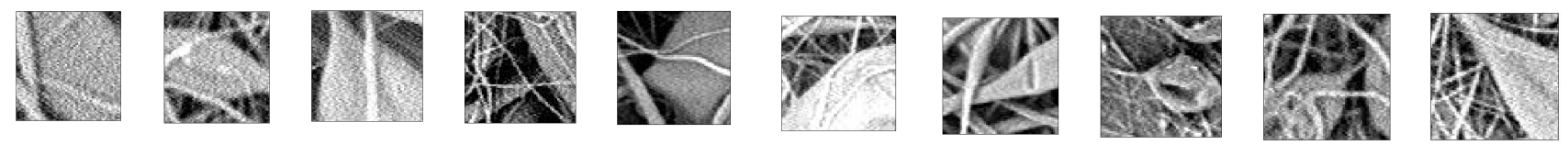

Reconstructed NH-NF SEM Nanopatches
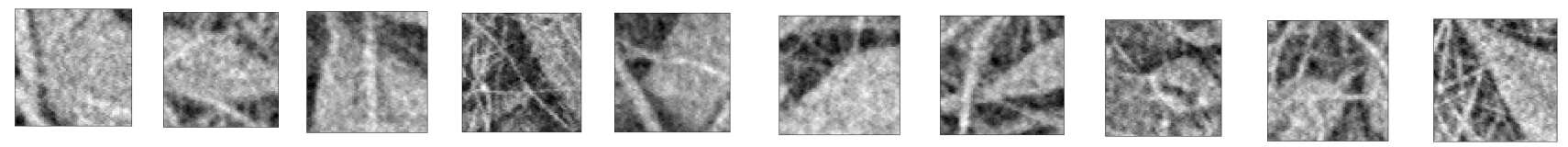

(b)

Fig. 7. (a) Representation of the 256-dimensional features vector extracted by a SEM NH-NF image (sized 64 x 64) via the proposed AE. (b) Examples of 10 reconstructed NH-NF images. 